\title{
The effect of stress and magnetostriction on the anisotropy of $\mathrm{CoNi} / \mathrm{Pt}$ multilayers
}

\author{
W.P. Van Drent ${ }^{1}$, M.D. Bijker ${ }^{*}$, J.C. Lodder \\ MESA Research Institute, University of Twente, P.O. Box 217, 7500 AE Enschede, The Netherlands
}

\begin{abstract}
An attempt is made to correlate the effective magnetic anisotropy of sputtered $\mathrm{CoNi} / \mathrm{Pt}$ multilayers to the average stress in the thin film. It was found that compressive film stress decreases the perpendicular magnetic anisotropy.
\end{abstract}

\section{Introduction}

Magneto-Optical (MO) multilayers are considered to be good candidates for future high-density MO recording $[1,2]$. From other research it is known (e.g. [3]) that there can be a considerable contribution of stress induced anisotropy to the total (perpendicular) anisotropy. To correlate this effective magnetic anisotropy to the average stress in the thin film, a series of sputtered $\mathrm{Co}_{50} \mathrm{Ni}_{50} / \mathrm{Pt}$ multilayers was prepared in which only the sputter pressure was varied. Each multilayer consists of a Pt seedlayer and a stack of $17 \mathrm{Co}_{50} \mathrm{Ni}_{50} / \mathrm{Pt}$ bilayers. The experimental details about the deposition are given elsewhere [4].

\section{Sample characterization}

The properties of the deposited samples are listed in Table 1. The layer thicknesses $t_{\mathrm{Seed}}, t_{\mathrm{CoNi}}$ and $t_{\mathrm{Pt}}$ were estimated from the product of sputter time and sputter rate. The bilayer thickness $t_{\text {bilayer }}$, measured by low angle XRD was found to be in good agreement with the projected layer thicknesses. The largest deviation was found for sample B (7\%). Furthermore the saturation magnetization $M_{\mathrm{s}}$ was calculated using the $\mathrm{CoNi}$ thickness only and found to be $1060 \pm 60 \mathrm{kA} / \mathrm{m}$ for all films. Magnetic torque measurements were performed at room temperature at an applied field of $1330 \mathrm{kA} / \mathrm{m}$. The effective anisotropies measured in this way, normalised to the magnetic volume, are also displayed in Table 1. To measure the film stress two different methods were applied: measurement of the surface curvature, and XRD measurements. The average internal film stress can be calculated

\footnotetext{
* Corresponding author. Email: m.d.bijker@eltn.utwente.nl; fax: $+31-53-430-9547$.

' Current address: Toyota Technology Institute, 2-12-1 Hisakata, Tempaku, Nagoya 468, Japan.
}

Table 1

Properties of the prepared samples

\begin{tabular}{lcccccc}
\hline Sample & $\begin{array}{l}P_{\mathrm{Ar}} \\
(\mu \text { bar })\end{array}$ & $\begin{array}{l}t_{\text {Seed }} \\
(\AA)\end{array}$ & $\begin{array}{l}t_{\mathrm{CoN} i} \\
(\AA)\end{array}$ & $\begin{array}{l}t_{\mathrm{Pt}} \\
(\AA)\end{array}$ & $\begin{array}{l}t_{\text {bilayer }} \\
(\AA)\end{array}$ & $\begin{array}{l}K_{\text {eff }} \\
\left(\mathrm{kJ} / \mathrm{m}^{3}\right)\end{array}$ \\
\hline $\mathrm{A}$ & 5 & 95 & 4.9 & 6.3 & 11.6 & -50 \\
$\mathrm{~B}$ & 8 & 95 & 5.0 & 5.8 & 10.1 & 7 \\
$\mathrm{C}$ & 11 & 94 & 5.1 & 5.7 & 10.7 & 137 \\
$\mathrm{D}$ & 30 & 95 & 5.0 & 6.1 & 11.5 & 324 \\
$\mathrm{E}$ & 40 & 95 & 5.0 & 6.0 & 11.1 & 376 \\
\hline
\end{tabular}

from the measured radii of curvature and the lattice spacings respectively. The strain free lattice spacing, $d_{0}[\AA]$, was determined in the following way: from the curvature measurements we know the pressure range at which the deposited multilayers are in a strain free situation, approximately $3.7 \times 10^{-2}$ [mbar]. The corresponding average lattice spacing as measured with the XRD was taken as $d_{0}$ and found to be $3.760 \pm 0.017 \AA$. Further experimental details about these methods can be found in Refs. [3,4]. Both methods deliver the results as depicted in Fig. 1a and b. Note that if the film stress is isotropic the equation given by Stoney [5] is used.

\section{Anisotropy model}

In order to correlate stress and magnetic anisotropy the following model was derived. For multilayers with interface anisotropy a semi-empirical formula [6] was used to determine the interface and bulk contributions:

$t_{\text {magn }} \cdot K_{\mathrm{eff}}=2 K_{\mathrm{S}}+K_{\mathrm{v}} \cdot t_{\text {magn }}$.

The volume anisotropy $K_{\mathrm{v}}$ consists of crystal anisotropy $K_{1}$ and demagnetization anisotropy $K_{\mathrm{D}}$. When a plane stress is homogeneously distributed throughout the magnetic layers of the multilayer, this will also add to the volume anisotropy by $K_{\text {stress }}$ which is defined as $K_{\text {stress }}=$ 

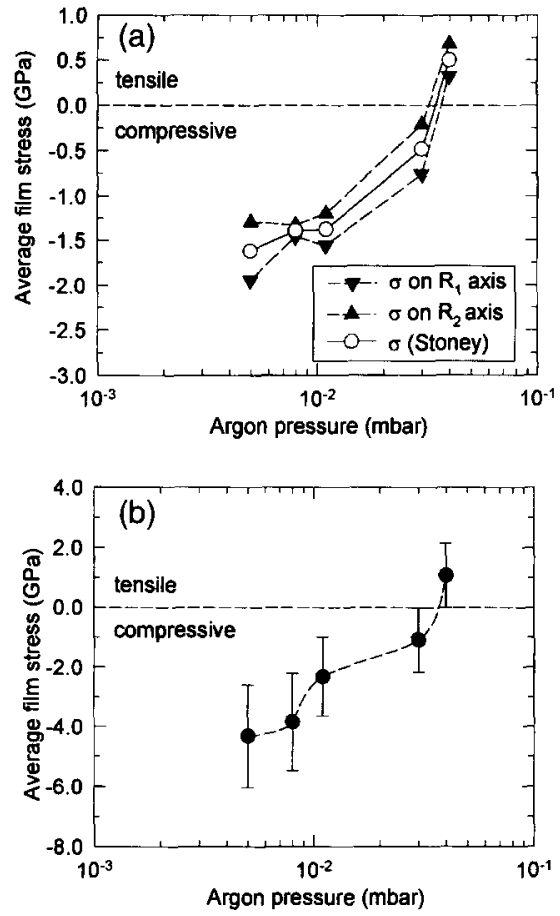

Fig. 1. (a) Stress along the $R_{1}, R_{2}$ axes and the stress calculated according to Stoney's equation. (b) Stress measured by XRD as a function of the argon pressure.

$-\frac{3}{2} \lambda_{\mathrm{S}} \sigma$, with $\lambda_{\mathrm{S}}$ the saturation magnetostriction and $\sigma$ the plane stress in the film. In this case a positive value for $\sigma$ denotes tensile stress. Together, the effective anisotropy is defined as:

$K_{\text {eff }}=2 K_{\mathrm{S}} / t_{\text {magn }}+K_{1}-\frac{1}{2} \mu_{0} M_{\mathrm{S}}^{2}-\frac{3}{2} \lambda_{\mathrm{S}} \sigma$.

This equation is schematically shown in Fig. 2. Now the saturation magnetostriction and the surface anisotropy can be calculated as being $\lambda_{\mathrm{S}}=-\frac{2}{3} \cdot \tan \alpha$ and $K_{\mathrm{S}}$ $=\frac{1}{2} t_{\mathrm{magn}}\left(P-K_{1}+\frac{1}{2} \mu_{0} M_{\mathrm{S}}^{2}\right)$ respectively, where $P$ and $\alpha$ are defined in Fig. 2.



Fig. 2. Schematical representation of Eq. (1). The dashed lines indicate the $x$ and $y$ axis. The linear fit with slope $\alpha$ crosses the $y$-axis at point $P$.

\section{Results and discussion}

High angle XRD experiments revealed that the $\mathrm{CoNi} / \mathrm{Pt}$ multilayers discussed here all had the fcc (111) structure. This corresponds to the fce structure found in the phase diagram of bulk $\mathrm{Co}_{50} \mathrm{Ni}_{50}$. Due to the fcc structure, $K_{1}$ is small and negative: $K_{1}=-108 \mathrm{~kJ} / \mathrm{m}^{3}$ for bulk $\mathrm{Co}_{50} \mathrm{Ni}_{50}$ [7]. Therefore for the following calculation it is assumed that the $K_{1}$ of the CoNi layers is $-108 \mathrm{~kJ} / \mathrm{m}^{3}$ over the whole argon pressure range. If we now perform the calculation according to Eq. (2), we arrive at $\lambda_{S}=-130 \times 10^{-6}$ and $K_{\mathrm{S}}=0.285 \mathrm{~mJ} / \mathrm{m}^{2}$ for the curvature measurements and at $\lambda_{\mathrm{S}}=-56 \times 10^{-6}$ and $K_{\mathrm{S}}=0.287 \mathrm{~mJ} / \mathrm{m}^{2}$ for the stress measurements based on XRD.

We observe that the surface anisotropy values of both methods are very similar. The $K_{\mathrm{S}}$ determined in this way compares well with results from magnetic layer thickness series from various authors (e.g. [8]). From this good match we conclude that the choice of the argon pressure at which the multilayer is in the stress-free state is justified.

Comparison of the saturation magnetostriction values reveals that the magnetostriction obtained by the surface curvature measurement is more than twice as large as that obtained from XRD results. A probable explanation is that during growth the thin film relaxes on a macroscopic scale, thereby reducing the curvature of the substrate. However, the stress measured with the XRD method gives a more realistic value of $\lambda_{\mathrm{S}}$. With XRD the average lattice spacing is measured, which represents the stress state within the crystallites of the layer. As the stress anisotropy is also generated on this local scale, the XRD values are thought to be more representative in this case.

In conclusion we can say that both methods indicate that the magnetic anisotropy changes from perpendicular to in-plane with increasing compressive film stress. Therefore compressive film stress, which increases with decreasing argon pressure, is considered to be unfavourable for (perpendicular) magneto-optic recording.

\section{References}

[1] S. Hashimoto, J. Appl. Phys. 75 (1994) 438.

[2] Q. Meng, W.P. Van Drent, J.C. Lodder, Th.J.A. Popma, J. Magn. Magn. Mater. 156 (1996) 296 (these Proceedings).

[3] S. Honda, N. Morita, J. Ago, Y. Tamura, M. Nawate, J. Magn. Magn. Mater. 121 (1993) 506.

[4] M.D. Bijker, W.P. Van Drent, J.C. Lodder, J. Magn. Magn. Mater. 48 (1995) 160.

[5] G.G. Stoney, Proc. Roy. Soc. A 32 (1909) 172.

[6] F.J.A. Den Broeder, H.C. Donkersloot, H.J.G. Draaisma, W.J.M. de Jonge, J. Appl. Phys. 61 (1987) 4317.

[7] R.M. Bozorth, Ferromagnetism (Princeton, NJ, 1951), pp. 277,570 .

[8] R. Krishnan, H. Lassri, M. Seddat, M. Porte, M. Tessier, Appl. Phys. Lett. 64 (1994) 2312. 